\title{
The implication of the osteolysis threshold and interfacial gaps on periprosthetic osteolysis in cementless total hip replacement
}

\author{
Hamidreza Alidousti ${ }^{a}$ and Neil W. Bressloff ${ }^{b}$ \\ a Department of Mechanical Engineering, Imperial College London, London, UK, SW7 2AZ \\ b Faculty of Engineering and the Environment, University of Southampton, Highfield, \\ Southampton, UK, SO17 1BJ
}

\section{Abstract}

2 Osteolysis around joint replacements may develop due to migration of wear particles 3 from the joint space into gaps between the interface bone and the implant where they 4 can accumulate in high concentrations to cause tissue damage. Osteolysis may appear 5 in various postoperative times and morphological shapes which can be generalized into 6 linear and focal. However, there are no clear explanations on the causes of such vari7 ations. Patients' degree of sensitivity to polyethylene particles (osteolysis thresholds), 8 the local particle concentration and the access route provided by the interface gaps 9 have been described as determining factors. To study their effects, a 2D computational

fluid dynamics model of the hip joint capsule in communication with an interfacial gap and the surrounding bone was employed. Particles were presented using a discrete phase model (DPM). High capsular fluid pressure was considered as the driving force for particle migration. Simulations were run for different osteolysis thresholds ranging from $5 \times 10^{8}$ to $1 \times 10^{12}$ particle number per gram of tissue and fibrous tissue generation in osteolytic lesion due to particles was simulated for the equivalent of ten postoperative years. In patients less sensative to polyethylene particles (higher threshold), osteolysis may be linear and occur along an interfacial gap in less than $5 \%$ of the interfatial tissue. Focal osteolysis is more likely to develop in patients with higher sensitivity to polyethylene particles at distal regions to an interfacial gaps where up to $80 \%$ of the interfatial tissue may be replaced by fibrous tissue. In these patients, signs of osteolysis may also develop earlier (third postoperative year) than those with less sensitivity who may show very minor signs even after ten years. This study shows the importance of patient sensitivity to wear particles, the role of interfatial gaps in relation to morphology and the onset of osteolysis. Consequently, it may explain the clinically observed variation in osteolysis development. 


\section{Introduction}

The relation between the presence of polyethylene wear particles and osteolysis around joint replacements is widely recognized (Schmalzried et al. (1992); Maloney et al. (1990); Willert et al. (1990)). Once wear particles are released from bearing surfaces into the joint space, they may be carried to the accessible periprosthetic spaces and tissues by the joint fluid (Schmalzried and Callaghan (1999)). However, the exact mechanism of osteolysis development is not completely understood. While some patients exhibit linear osteolysis, others develop focal ones and some remain immune to osteolysis despite excessive wear generation in their prosthetic joint (Jasty et al. (1997)). In addition, the onset of osteolysis can vary between one to seven years (Tanzer et al. (1992)). Although many factors are likely to contribute to these variations in osteolysis development, patient's sensitivity to polyethylene, local particle concentration which relates to particle migration to the interfacial tissue by means of interface gaps could be determining factors.

It has been shown that, if particle concentration reaches a certain threshold in periprosthetic tissue, osteolysis may develop. Revell et al. (1997) and Kobayashi et al. (1997) showed that sites containing more than the critical value of approximately 1 $\times 10^{10}$ particles per gram of tissue had developed focal osteolysis. However, Elfick et al. (2003) reported a lower threshold of $1 \times 10^{9}$ particles per gram of tissue for osteolysis generation. Koseki et al. (2005) showed that the particle concentration in focal lesions $\left(2.10 \times 10^{9}\right.$ particles per gram of tissue $)$ was significantly greater than the linear ones $\left(2.91 \times 10^{8}\right.$ particles per gram of tissue). These findings show that once the threshold is reached, the bone tissue is replaced with fibrous tissue with lower permeability which may encourage more particles to flow in that region. In addition, Ise et al. (2007) observed a correlation between the ratio of osteolysis in radiographic images and patient sensitivity to polyethylene particles. These findings suggest that the biological threshold for osteolysis generation may be patient specific and that osteolysis generation in the long term could be dependent on this threshold.

A number of studies have investigated particle migration to the periprosthetic tissue to some extent. Bobyn et al. (1981) observed that polyethylene debris $\left(1 \times 10^{8}\right.$ particles twice a week) injected in the joint migrated easily along the smooth surfaces of cylindrical implants implanted into the distal femur and proximal tibia of dogs. They observed that, during this time, particles penetrated the intratrabecular spaces up to approximately $2 \mathrm{~mm}$. The findings of von Knoch et al. (2000) showed that the path of least resistance for distal migration is provided by the interfacial gaps and not through the porosity of the bone and fibrous tissues.

The above studies considered only the end-stage of osteolysis and do not describe how particle-induced osteolysis develops and expands during the lifespan of an implant. 
The current study aims to investigate how osteolysis expansion and development may be affectd by an osteolysis threshold and an existing interfacial gap which may facilitate particle transportation. A computational fluid dynamics (CFD) model which simulates particle migration and entrapment in periprosthetic gaps and bone tissue as well as fibrous tissue generation was employed.

\section{Method}

The 2D geometry of the model was based on a realistic 3D model of a femur reconstructed from a computerized tomography (CT) scan implanted with a cementless femoral stem (Summit stem, DePuy, Warsaw USA) and the surrounding joint capsule. The 2D geometry was generated by cutting through the symmetric axis of the implant on the coronal plane (c.f. Figure 1 a). The boolean operation for implantation and 2D geometry generation were carried out in Solidworks (Dassault Systemes, Velizy, France). The model geometry was meshed in GAMBIT (Ansys Inc.) using structured (gap region) and unstructured (capsule and bone regions) quadrilateral cell volumes and then imported into the commercial CFD software FLUENT (version 12.00, Ansys Inc.) for analysis.

The meshed geometry of the model is shown in Figure 2. The geometry was meshed with approximately $10 \mathrm{k}, 21 \mathrm{k}, 42 \mathrm{k}$, and $140 \mathrm{k}$ cells for mesh convergence studies. For each of these meshes, different combinations of the core and boundary layer meshes (which included varying the height of the first layer and the total number of layers) were generated. Mesh convergence studies demonstrated that the $42 \mathrm{k}$ mesh with an appropriately sized boundary layer and core meshes (c.f. Figure 2) showed less than $5 \%$ variation in fluid velocity in regions of high flow gradient, relative to the finest mesh. Therefore, this mesh was deemed appropriate for this study.

The capsule and gap regions were modelled as free fluid continua, the bone was represented as a porous medium and the implant wall was described as a rigid wall. Simulations were steady state and did not incorporate the fluid pumping mechanism caused by micromotion between the implant and bone. High capsular fluid pressure was considered to be the driving force for particle transportation (Alidousti et al. (2014); Schmalzried et al. (1997); Robertsson et al. (1997)). Fluid pressure inside the capsule was generated by a pressure inlet boundary defined on the soft tissue wall of the capsule. High capsular pressure was considered to be $60 \mathrm{kPa}$ (Hendrix et al. (1983)). The pressure over the porous bone region was assumed to be zero with respect to the capsule (i.e. $0 \mathrm{kPa}$ ). This was achieved by defining a pressure outlet on the endosteal and periosteal surfaces of the bone (c.f. Figure 1 b). This boundary condition may represent the fluid drainage through many embedded venous sinuses in proximal femur which leave the bone through a great number of openings 
on the external surfaces of the femur in medial, lateral and superior regions (Churchill et al. (1992)). In addition, one source can extend to supply other regions when there is impairment to another. This is indeed the case for an implanted femur in which the femoral cavity is filled with the implant stem and the blood supply is provided from the external periosteal surface. In fact, it has been shown that two thirds of the total blood supply and drainage in the bone surrounding the implant is carried out by periosteal, epiphyseal and metaphyseal arteries which penetrate the bone through external periosteum layer (Bridgeman and Brookes (1996)). Rhinelander (1972) has also shown that the greater portion of blood in diaphysis is drained from the periosteal surface rather than endosteal. In the cortical bone, it has been shown that the blood supply entering the Haversian system may also exit the system through the periosteal blood supply (Cooper et al. (1966); Montgomery et al. (1988)). These observations are further supported by the fact that intravascular pressure drops from about $8 \mathrm{kPa}$ to around $2 \mathrm{kPa}$ from endosteum to periosteum surfaces (Brookes (1971)) which indicates the fluid drainage from the centre the periphery of the bone as simulated in the current study. In addition, it would be reasonable to assume that there is an equilibrium between fluid flux entering the bone region uniformly from the lymphatic and vascular system and the fluid flux that leaves the bone uniformly through the same system. Therefore, blood supply and drainage does not create any permanent or regional fluid sinks or sources which needs to be accommodated in the model. The excessive fluid entering the bone from the joint capsule may also leave this region through the vascular system. However, as mentioned above, in an implanted femur, the vascular flow may carry this particle-laden fluid to the periphery and the outer surface of the bone. The zero pressure outlet boundary condition used in the current study may provide a simplified representation of this system by directing particle-laden fluid out of system in the same direction as the vascular system does.

Based on arthrographic studies (Hendrix et al. (1983); Cone et al. (1983)), in which it can be seen that the contrast agent injected to the joint capsule immediately finds its way to the interfacial gaps and osteolytic lesions in communication with them, it was assumed that the synovial fluid occupies the interfacial gaps and it can easily flow to the interface. The fluid flowing in the capsule, gap and the bone was, therefore, modelled with the properties of liquid water with constant viscosity and density of $0.001 \mathrm{~kg} / \mathrm{ms}$ and $998.2 \mathrm{~kg} / \mathrm{m} 3$, respectively. Fluid flow in the capsule and gap - where viscous effects are important - was described by the Navier-Stokes equations and laminar viscous flow was assumed throughout. Fluid flow in the bone - where viscous effects are not significant and permeability governs the diffusive flow - was defined by Darcy's equation which was added as a sink in the momentum equations given by:

$$
\rho\left[\frac{\partial \mathbf{v}}{\partial t}+\mathbf{v} \cdot \nabla \mathbf{v}\right]=-\nabla P+\nabla \cdot(\mu \nabla \mathbf{v})-\left(\frac{\mu}{k}\right) \mathbf{v}
$$


where, $\mathbf{v}$ is the fluid velocity $(\mathrm{m} / \mathrm{s}), \rho$ is the fluid density $\left(\mathrm{kg} / \mathrm{m}^{3}\right), \mathrm{t}$ is time $(\mathrm{s}), P$ is the fluid pressure $(\mathrm{Pa}), \mu$ is the dynamic fluid viscosity $(\mathrm{kg} / \mathrm{m} . \mathrm{s})$ and $\mathrm{k}$ is the porous medium permeability $\left(\mathrm{m}^{2}\right)$.

It should be noted that in the bone region the viscous terms of Eqn.1 become insignificant due to the very small velocity gradient across the bone region as the result of low permeability effect. This means that Eqn.1 reduces to Darcy's equation and the fluid in the bone region is governed almost entirely by the Darcy's law. Therefore, Eqn.1 accommodates viscous and porous effects separately at the appropriate location in the model.

Darcy's law have been previously employed to simulate the flow of polymethylmethacrylate and interstitial fluid through cancellous and cortical bone (Johansson et al. (2009); Beaudoin et al. (1991)). In addition, it has been experimentally shown that Darcy's law remains valid for laminar flows in bone below the Re value defined by Eqn.2 (Arramon and Nauman (2000)):

$$
R e=4 \frac{\rho \bar{V}}{\mu S_{v}}
$$

where, $\bar{V}$ is the fluid velocity through the porous medium and $S_{v}$ is specific surface of for porous bone (Arramon and Nauman (2000)). Using the above equation, an $\operatorname{Re}=4.6$ was calculated based on the maximum fluid velocities entering the bone in the vicinity of the gap. However, the Re values in the gap region of current model, calculated by using the gap width as the characteristic length scale, are an at least 35 times smaller than this threshold $\mathrm{Re}=4.6$. Therefore, the current model remains in the valid and laminar flow regime to use Darcy's law.

Bone permeability of $k=1.00 \times 10^{-14} \mathrm{~m}^{2}$ was chosen based on a computational representation of an experiment in which Simkin et al. (1985) measured the hydrostatic resistance of metaphyseal bone to fluid injection. This permeability may be referred to as the 'effective permeability' since it takes into account the effect of bone marrow, which includes non-fluid constituents such as blood vessels, fat cells and the loose connective framework that maintains the mechanical integrity of the marrow (Bryant et al. (1989)), and the external cortical shell that encapsulates the periprosthetic cancellous bone. The calculated effective permeability lies above the averaged measured permeability of cancellous bone with no marrow $\left(k=1.00 \times 10^{-10} \mathrm{~m}^{2}\right.$ (Nauman et al. (1999); Hui et al. (1996); Thompson et al. (2004); Kohles et al. (2001); Pakula et al. (2008))) and below the permeability of cortical bone at vascular level $\left(k=6.3 \times 10^{-15} \mathrm{~m}^{2}\right.$ Zhang et al. (1998)). The process of defining such a permeability value is explained in details in Appendix A. All the computational cells in the bone region were assigned this permeability value initially. Whilst the steady state governing equations were solved, unsteady tracking of particles in time was incorporated as described below. The model 
included a short interfacial gap with $5 \mathrm{~mm}$ length and $30 \mu \mathrm{m}$ width. The choice of gap dimension was described in detail by Alidousti et al. (2011).

A time step of $0.002 \mathrm{~s}$ showed sufficient accuracy with less than $5 \%$ variation in particle trajectories compared to time steps as low as $0.0002 \mathrm{~s}$. The simulations comprised 5000 time steps representing ten post-operative years by incrementally injecting ten years worth of wear particles into the model during this computational time.

Table 1 shows the setup for the simulations. The default under-relaxation factors for convergence were used and the convergence criterion for mass and velocity residuals was set to $1 \times 10^{-7}$. Standard pressure discretization was employed since it is the method recommended by FLUENT for models with porous media (Fluent (2015))

The rate of wear generation depends on many parameters such as patient activity, the femoral head size, relative position between the head and cup etc. A simple calculation on the result of an in vitro wear simulation on polyethylene-on-metal bearings by Shorez et al. (2008) indicated that approximately $6.7 \times 10^{10}$ particles are resealed into the joint space per year on average. This rate of wear generation was chosen for the current study. Therefore, 13.4 million particles were injected to the flow in each computational time step while previously injected particles were tracked.

\section{Particle modelling}

To simulate wear particle migration, spherical particles with the diameter of $1 \mu \mathrm{m}$ and the density of polyethylene $(935 \mathrm{~kg} / \mathrm{m} 3)$ were incorporatd into the model as a discrete phase within the continuous fluid phase (Fluent (2015)). The choice of particle size was based on the fact that approximately $90 \%$ of the particles in cells from total hip replacement patients were just less than $1 \mu \mathrm{m}$ (Revell et al. (1997); Koseki et al. (2005); Benz et al. (2001)). Momentum was transferred to particles as they passed through each control volume, and the effect of particles on the continuum was incorporated in the model by a two-way coupling algorithm provided by FLUENT. There were no particle-to-particle interactions. Particles were introduced to the continuous flow as "parcels" (to reduce computational time) by means of discrete injection points located at the gap entrance (Figure $1 \mathrm{~b}$ ). For more detailed description of particle modelling and injection refer to Alidousti et al. (2014).

Permeability of the periprosthetic tissue may change due to particles clogging the interface tissue and reducing its porosity. Changes to the local bone porosity due to particle clogging was defined by:

$$
\phi_{\text {new }}=\phi_{0}-\frac{V_{p}}{c_{v}} .
$$

where $\phi_{\text {new }}$ is new porosity, $\phi_{0}$ is initial porosity, $c_{v}$ is the local computational cell volume and $V_{p}$ is the volume occupied by the instantaneous number of particles in that 
cell volume. The initial porosity $\phi_{0}$ value was calculated based on the effective permeability using the Kozeny-Carmen model (Arramon and Nauman (2000)) shown by Eqn. 4 which relates porosity to permeability for cancellous bone. Although KozenyCarmen model was originally developed for cancellous bone only, it is based on hydraulic radius-based capillaric tubes with an added tortuosity factor that accounts for bends in a random pore structure such as that of periprosthetic tissue adjacent to an interfacial gap. Therefore, it provided the best available tool and first approximation to calculate the new permeability for the clogged bone $\left(k_{n e w}\right)$ as a function of the new porosity $\phi_{\text {new }}$ :

$$
k_{\text {new }}=\frac{c \phi_{\text {new }}^{\alpha}}{S_{v}\left(\phi_{\text {new }}\right)^{2}},
$$

where $c$ and $\alpha$ are correlation coefficients. Throughout the simulations, the permeability of the tissue was calculated and updated by Eqn. 4 according to the instantaneous number of particles in each computational cell.

There is no data available in the literature describing particle clogging in the periprosthetic bone-marrow system during postoperative periods. However, it has been clinically observed that particle concentration is highest in close proximity of the interface and decreases further from the interface when there is a continuous supply of particles (Bobyn et al. (1995); Kwong et al. (1992); Hirakawa et al. (1996); Margevicius et al. (1994). Such a phenomenon is also seen in particle filtration studies in geological material (Civan et al. (1989)).

Therefore, an empirical parameter for particle clogging was developed to produce the above clinical observation for a ten year period during the computational simulation time. In order to achieve this, a parameter called clogging factor was defined $\left(c_{f}\right)$. $c_{f}$ defined the probability of the particles being trapped or stopped in the periprosthetic tissue. To achieve this, particles were tagged in each time step by randomly generated numbers between zero and one. Particles in the bone region which were tagged in a certain range were assigned a zero velocity. This made it possible to control the percentage of particles that randomly clogged the bone region. Plots showing particle concentration along a profile line located in the mid-gap region perpendicular to the interface for clogging factor values of $0 \%, 30 \%$ and $50 \%$ are shown in Figure 3. For clogging factors larger than $50 \%$, particle penetration was effectively blocked and only a few particles penetrated the bone beyond $50 \mu \mathrm{m}$ from the interface. In contrast, clogging factors less than $50 \%$ prevented particle accumulation taking place at the interface as seen in Figure 3. This parametric analysis showed that, for a wear generation period of ten postoperative years, $c_{f}=50 \%$ produced the most realistic particle distribution at the interface closely resembling clinical observation. This value was used to simulate clogging in the current simulations. 


\section{Fibrous tissue generation simulation}

In studies in which the particles in the fibrous tissue occupying osteolytic lesions were counted and the osteolysis threshold $\left(o_{t}\right)$ was established (Kobayashi et al. (1997); Koseki et al. (2005); Elfick et al. (2003); Revell et al. (1997)), the data was presented in terms of the particle number per gram of tissue. To make this value appropriate for the current study, the particle concentration has to be presented in terms of tissue volume. Assuming a consistent particle distribution throughout the tissue and a density of $1500 \mathrm{~kg} / \mathrm{m}^{3}$ for periprosthetic tissue, $1 \mathrm{~g}$ of periprosthetic tissue consists of a cube with a volume of $6.667 \times 10^{-7} \mathrm{~m}^{3}$ and dimensions of approximately $8.7 \times 8.7 \times 8.7$ $\mathrm{mm}$ in which the one billion particles are evenly distributed. However, it is possible to calculate the threshold for smaller volumes of tissue using linear interpolation. For example, this threshold reduces to 750 particles for a cube with dimensions of $10 \times 10$ $\times 50 \mu \mathrm{m}$ as shown in Figure 4 .

By assuming a depth of $50 \mu \mathrm{m}$ for the model (close to the width of an interfacial gap), it is possible to use the same linear interpolation to calculate the corresponding osteolysis threshold for each of the computational cell volumes in the model. The instantaneous number of particles in each computational cell volume in the bone region was calculated in each time step. If the number of particles in a volume cell rose above the specified threshold defined for each cell, fibrous tissue permeability value of $k=1 \times 10^{-11} \mathrm{~m}^{2}$ (Prendergast et al. (1997)) was assigned to that computational cell, thus simulating the effect of osteolysis. To study the effect of osteolysis threshold in the current simulations, $o s_{t}$ was varied from $5 \times 10^{8}$ to $1 \times 10^{12}$ particle number per gram of tissue according to the aforementioned studies.

It is important to clarify that, in the current model, the shape and the extent of the fibrous tissue is dependent on the accuracy of particle trajectories. If the mesh density is sufficiently fine to accurately capture the particles trajectories, then the mesh density may only affect the spacial resolution of the fibrous tissue lesions. This is due to the fact that the fibrous tissue regions in the model are identified by those cell volumes that have their permeability increased due to high particle concentration. In the current study, the mesh size in the bone region in vicinity of the gap was approximately 30 $\mu m^{2}$ for the largest cell volumes (c.f Figure 2). This cell volume size in the bone region is in fact smaller than the average pore size in the cancellous bone (300-1000 $\left.\mu \mathrm{m}^{2}\right)$ or an osteon (100-150 $\mu^{2}$ in diameter) in cortical bone (Cowin (2000)). Therefore, the current mesh density may provide sufficient spatial resolution to capture fibrous tissue generation in the bone tissue. 


\section{Data analysis}

The data was analyzed by contour plots of periprosthtic tissue showing regions of bone and generated osteolytic fibrous tissue as a result of particle presence. The interface tissue was defined by an area with a $1.5 \mathrm{~mm}$ width which extended distal to the interfacial gap (Figure 5 and Figure 6). The width and length of this area were chosen based on the maximum fibrous tissue penetration observed in the preliminary models. The ratio of fibrous tissue to bone in the periprosthtic regions was calculated by measuring the area of each tissue type in proximal, middle and distal regions of the gap. The total area for each of these three regions was equal (Figure 6) and the area was calculated accurately by counting the number of pixels in each region. Pixel counting was carried out by using Matlab Image Processing Toolbox (MathWorks, Inc., Natick, Massachusetts, United States Release 2015a). Using the calculated areas of each tissue type, the ratio of fibrous tissue to bone in each region of interface tissue was calculated.

\section{Results}

It was seen that, as particles entered a region, clogging and permeability reduction occured. This led to slower particle penetration resulting in particle accumulation and high concentrations. Once the osteolysis threshold was reached due to accumulation, tissue permeability increased to that of fibrous tissue causing more particles to flow to that region. This loop repeated as regions with increased permeability (fibrous tissue) penetrated into periprosthetic bone. Therefore, particles penetrated further into the bone through distinguishable paths of least resistance generated by the fibrous tissue (Figure 5). This development and progression of fibrous tissue around the interface gap for three different thresholds - low $\left(o s_{t}=5 \times 10^{8}\right)$, medium $\left(o s_{t}=1 \times 10^{9}\right)$ and high $\left(o s_{t}=1 \times 10^{10}\right)$ - are shown in Figure 5 for three, six and ten post-operative years. This figure shows that threshold for osteolysis, or in other words patient sensitivity to polyethylene particles, had a direct impact on the fibrous tissue shape and expansion. In cases run with $o s_{t}=5 \times 10^{8}$ and $1 \times 10^{9}$ particles per gram of tissue (Figure 5 a and $b$ ), periprosthetic tissue was not significantly affected in the first operative year (not shown here). In these cases, the fibrous tissue started appearing from the second postoperative year and then it progressed through bone tissue as time elapsed. By the tenth postoperative year, it had penetrated the bone approximately $0.6 \mathrm{~mm}$ in regions located along the gap and approximately $1.5 \mathrm{~mm}$ in regions located at the bottom of the gap. However, for $o_{t}=1 \times 10^{10}$ particles per gram of tissue (Figure $5 \mathrm{c}$ ), fibrous tissue only developed in very small and isolated regions located at the gap entrance and bottom by the sixth postoperative year. It was only by the tenth year that distinctive but small regions of fibrous tissue were observed. Nevertheless, the pattern of fibrous 
tissue generation tended to be linear in this case. As $o s_{t}$ increased to $5 \times 10^{10}$ particles per gram of tissue and any value above, no fibrous tissue generation occurred in the model and only a permeability reduction was observed a a result of particle clogging the periprosthetic tissue.

A quantitative representation of Figure 5 is shown in Figure 6 where the ratio of fibrous tissue to bone at the interface tissue in proximal, middle and distal regions to the gap are shown. Figure 6 shows that, in high and moderate polyethylene sensitivity $\left(o s_{t}=5 \times 10^{8}\right.$ and $1 \times 10^{9}$ particles per gram of tissue, respectively), a greater portion of the periprosthetic tissue was occupied by osteolytic fibrous tissue as time elapsed. However, this osteolysis progression was approximately four times faster at distal regions of the gap between the 6th and 10th postoperative years where up to $80 \%$ of the distal interfacial tissue was occupied by fibrous tissue. Figure 6 also shows that, in patients with low sensitivity to polyethylene particles $\left({ }^{\circ} s_{t}=1 \times 10^{10}\right.$ particles per gram of tissue), who showed linear osteolysis generation, less than $5 \%$ of interface tissue was affected by osteolysis.

\section{Discussion}

The current study indicates that osteolysis threshold may play an important role in the onset and morphology of osteolysis development around a joint replacement implant. It was shown in patients less sensitive to polyethylene particles (high osteolysis threshold), in the presence of an interfacial gap, linear and less extensive osteolysis may occur along the gap in only late postoperative stages, and in patients more sensitive to polyethylene, focal and extensive osteolysis may develop distal to these gaps. The models show how progressive fibrous tissue generation at the interface may provide a pathway for particles to penetrate deep into the tissue and how, in turn, a constant supply of particles causes further expansion of these regions in osteolytic lesions, particularly when a patient is sensitive to polyethylene particles.

There are a number of clinical observations that relate to the current simulations and also can be used as an indirect verification of the their outcome. Firstly, the pattern of particle penetration seen in the current study is similar to that described by Schmalzried et al. (1992). They observed that fibrous tissue stroma running like a stream through the periprosthetic tissue providing channels or routes for particle penetration which leads to expansion of the effective joint space. They suggested that the difference between focal and linear osteolysis may be related to the concentration and distribution of particles in the periprosthetic tissue. However, the simulations in the current study have shown that patient sensitivity to polyethylene particles as well as particle accumulation at the bottom of the interfacial gaps may also play a role in differentiating linear or focal lesions. Secondly, the models here have shown 
that conditions for osteolysis generally start to appear between the second and third postoperative years. This temporal prediction is in accordance with clinically observed osteolysis previously shown (Tanzer et al. (1992); Donnelly et al. (1997); Maloney et al. (1990); Zicat et al. (1995). Thirdly, the critical osteolysis threshold values have been described to be between $1 \times 10^{9}$ (Elfick et al. (2003)) and $1 \times 10^{10}$ (Kobayashi et al. (1997) per gram of tissue. The results also showed that osteolysis progression patterns also change in the vicinity of the these thresholds. Fourthly, there are patients with high rates of wear generation who show no signs of osteolysis (Jasty et al. (1997)). One possible explanation for this phenomenon is that the biological sensitivity to particles in these patients is low, i.e. high osteolysis threshold. In fact, Ise et al. (2007) observed a correlation between the ratio of osteolysis in radiographic images and patient sensitivity to polyethylene particles. They showed that, the point at which osteolysis occurs and the speed of its development varied in each individual. The speed of progression was faster for patients who were more sensitive to polyethylene particles. The results also showed that osteolysis threshold, which may represent the degree of biological sensitivity, plays an important role in the temporal and regional progression of osteolysis and can predict such variation in patients. Fifthly, the pattern of fibrous tissue observed in the current study is in agreement with the prediction of previous results using similar methodology (Alidousti et al. $(2014,2011)$ ) which showed higher flow rates of particles in the distal regions of an interfacial gap. Furthermore, a width of $1.5 \mathrm{~mm}$ osteolysis development seen in the current study, is very close to what Bobyn et al. (1995) observed. They injected a quantity of polyethylene particles $\left(2 \times 10^{9}\right)$, similar to the current study, into rabbit joint and observed osteolytic lesions, some of which were expansile and $1.5 \mathrm{~mm}$ wide, which appeared as radiolucencies along smooth surfaces of implants in radiographs.

In Figure 5 c, the fibrous tissue was developed slightly away from the interface, but not at the interface. This is due to the fact that fibrous tissue generation initially occurs at the penetrating particle front which encourages more particles flow to this region of higher permeability. This subsequently increases particle accumulation and the chance of fibrous tissue generation at this penetrating front compared to those regions behind. As this highly concentrated front moves away from the interface, regions closer to the interface may turn into/remain bone because of their lower particle concentration. This may simulated the healing process which may occur in vivo as particles spread out throughout the tissue causing lower concentrations. In the case of Figure $5 \mathrm{c}$, the majority of resorption takes place between $t=6$ to 10 years. Although the entire interface tissue contains particles, the highly concentrated regions with concentrations above the threshold are located away from the interface at the instance of $t=10$ years. It should also be noted that penetration of particles is less in the case of higher osteolysis threshold (Figure 5 c) due to the absence of fibrous tissue to provide transport route 
for particles.

In should also be noted that the bone tissue around an implant may experience increased permeability due to stress shielding or ageing or decreased permeability due to excessive loading leading to denser bone. Therefore, bone permeability change due to these factors may not be uniformly distributed around an implant. Simulating these scenarios may provide valuable information. However, it requires further investigation and a parametric analysis which are outside the scope of the current study. Nevertheless, the current model may still represent those patients who preserve the bone quality, at least in the vicinity of a short gaps such as the one simulated in the current study.

There are a number of limitations associated with the current study. Firstly, the number of particles considered in the current work were for a normal functioning implant. However, not all generated particles enter or have access to interfacial gaps. Estimating the exact number of particles flowing into an interfacial gap, which depends on many factors such as the number of gaps, the extent and volume of the effective joint space etc, is difficult. It is possible that, in reality, less particles than generated in a normally functioning hip have access to a gap which sets the lower limit of wear access to the interface. On the other hand, it has been shown that the wear generation in problematic THRs can be seventy fold more than normally functioning implants Dowd et al. (2000), which sets the upper limit of wear generation. Therefore, it is possible to assume that the number of particles injected into the gap region in the current work is in the realistic range of particle access to the interface between these lower and the upper limits. The number of particles that have access to the interface may influence the temporal characteristics of particle clogging and the extent of fibrous tissue generation, but it does not influence the results qualitatively.

Secondly, only one particle size is considered in the current study while there may be a distribution of particle size in the joint space which may have an effect on tissue clogging and fibrous tissue generation. Large particles may more readily clog the tissue while small particles (for the same number of particles per unit volume) would occupy a much smaller volume fraction with less clogging effect. However, they may result in a higher particle concentration and an increased chance of osteolysis development. The trade-off between the clogging effect of large particles and high concentration caused by smaller particles is a phenomenon which has to be addressed in a future work. Future models could be used to establish which particle size ranges are more likely to cause osteolysis through either mechanism. The future models could also study the effect of a heterogeneous particle size distribution on tissue clogging and fibrous tissue generation with consideration of biologically active size ranges described in the literature.

Thirdly, there are drainage mechanisms to clear some of the particles from the periprosthetic tissue (Noble et al. (1983); Urban et al. (2000); Jell et al. (2006)). How- 
ever, no drainage mechanism is included in the current work. Despite the existence of such mechanisms, a large number of particles accumulate at the interface (Manley et al. (2002)). This shows the insufficiency of this mechanism to clear significant number of particles from the tissue. Therefore, models run without such mechanisms may still represent in vivo conditions.

Fourthly, the current study only includes short gaps. As particle mean concentration is less for longer gaps (as shown by Alidousti et al. (2014)) due to their wider spread, tissue clogging and permeability reduction may be less. Therefore, conditions for development may be generated later compared to shorter gaps. However, this does not change the outcome qualitatively. Fifthly, gap displacements are not included in the simulations in the current study. However, Alidousti et al. (2014) have shown that capsular pressure, rather than gap displacement is the main driving force to pump particles into the interfacial gap. Sixthly, a long postoperative time of ten years was simulated by incrementally injecting ten years worth of particles in total to the model and introducing a clogging factor which would result in a realistic particle distribution as observed clinically. Although this is a gross approximation, simulations of this kind have not been performed before and they provide the foundations for developing a better understanding of the causes of osteolysis.

Finally, the constitutional equations for this study could have be driven from mixture theory or its simpler form, biphasic theory, to include load-induced flows in the system. However, it has been shown that load-induced flows are incapable of transporting or even moving particles around a tibila plate (Yuan X et al. (2000)). This could be due to the fact that the net flow of fluid caused by pressure difference due to mechanical loading is reversed back as soon as the load is removed (Knothe (2003)) leaving particles in their original position. This shows that a model developed solely based on mixture or Biot's theory is not capable of simulating particle transportation in the tissue. Since particle migration is commonly seen, it confirms the existence of other particle transport mechanisms such as high capsular fluid pressure, which can be considered the most effective mechanism of particle transportation (Alidousti (2012)). On the other hand, it is possible to show that load-induced flows have significantly lower velocity, and therefore momentum, to have any significant effect on particle transportation compared to those flows generated by high capsular fluid pressure. Load-induced flows in poroelastic models of bone under physiological loading do not exceed the velocity of approximately $0.2 \mu \mathrm{m} / \mathrm{s}$ - even in a bone which contains fibrous tissue and has higher permeability than normal bone (Prendergast et al. (1997)). However, the smallest velocity measured in the bone in the vicinity of the gap in any of the current models was at least an order of magnitude larger than this value. In fact, the fluid velocity of $17 \mathrm{~mm} / \mathrm{s}$ in the pressurised bone in the model here is very close to the value of $20 \mathrm{~mm} / \mathrm{s}$ estimated by Fahlgren et al. (2010) in rat bone pressurised to $90 \mathrm{kPa}$ 
470 This could be taken as an indirect validation of the velocity values computed in the 471 current work and as indication that high capsular fluid pressure is significantly more 472 effective in transporting particles due to high velocity flows. Therefore, the assump473 tion of permeable but nondeformable porous bone is reasonable for the purpose of this 474 study which is to simulate particle transport in periprosthetic tissue rather than bone 475 mechanosensation which could be affected by load-induced flows.

476 In summary, the results showed that there is a relationship between the osteolysis 477 threshold and the temporal and regional development of fibrous tissue in osteolytic 478 regions. They showed how high biological sensitivity to polyethylene particles along 479 with the existence of an interfacial gap can have a synergistic effect to produce focal 480 osteolysis at the implant interface. They also showed that patients who are less sensitive to polyethylene particles are more likely to develop linear osteolysis if a gap exists at the interface. Finally, this study highlights the importance of fixation to seal the interface from the joint fluid to prevent long-term loosening due to osteolysis. 


\section{References}

Alidousti, H. (2012). Periprosthetic fluid flow, particle distribution modelling and the implications for osteolysis in cementless total hip replacements. $\mathrm{PhD}$ thesis, University of Southampton.

Alidousti, H., Taylor, M., and Bressloff, N. W. (2011). Do capsular pressure and implant motion interact to cause high pressure in the periprosthetic bone in total hip replacement? Journal of biomechanical engineering, 133(12):121001.

Alidousti, H., Taylor, M., and Bressloff, N. W. (2014). Periprosthetic wear particle migration and distribution modelling and the implication for osteolysis in cementless total hip replacement. Journal of the Mechanical Behavior of Biomedical Materials, $32: 225-244$.

Arramon, Y. and Nauman, E. A. (2000). The intrinsic permeability of cancellous bone. In Cowin, S., editor, Bone mechanics. Informa Healthcare, New York, second edition.

Beaudoin, A. J., Mihalko, W. M., and Krause, W. R. (1991). Finite element modelling of polymethylmethacrylate flow through cancellous bone. Journal of biomechanics, 24(2):127131-129136.

Benz, E. B., Federman, M., Godleski, J. J., Bierbaum, B. E., Thornhill, T. S., and Spector, M. (2001). Transmission electron microscopy of intracellular particles of polyethylene from joint replacement prostheses: size distribution and cellular response. Biomaterials, 22(21):2835-42.

Bobyn, J. D., Jacobs, J. J., Tanzer, M., Urban, R. M., Aribindi, R., Sumner, D. R., Turner, T. M., and Brooks, C. E. (1995). The susceptibility of smooth implant surfaces to periimplant fibrosis and migration of polyethylene wear debris. Clinical Orthopaedics and Related Research, (311):21-39.

Bobyn, J. D., Pilliar, R. M., Cameron, H. U., and Weatherly, G. C. (1981). Osteogenic phenomena across endosteal bone-implant spaces with porous surfaced intramedullary implants. Acta Orthop Scand, 52(2):145-53. 
Bridgeman, G. and Brookes, M. (1996). Blood supply to the human femoral diaphysis in youth and senescence. J Anat, 188 ( Pt 3):611-21.

Brookes, M. (1971). The blood supply of bone. Butterworth \& Co.

Bryant, J., David, T., Gaskell, P., King, S., and Lond, G. (1989). Rheology of bovine bone marrow. ARCHIVE: Proceedings of the Institution of Mechanical Engineers, Part H: Journal of Engineering in Medicine 1989-1996 (vols 203-210), 203(28):7175.

Churchill, M. A., Brookes, M., and Spencer, J. D. (1992). The blood supply of the greater trochanter. J Bone Joint Surg Br, 74(2):272-4.

Civan, F., Knapp, R. M., and Ohen, H. A. (1989). Alternation of permeability by fine particle processes. Journal of Petroleum Science and Engineering, 3:65-79.

Cone, R. O., Yaru, N., Resnick, D., Gershuni, D., and Guerra, J. (1983). Intracapsular pressure monitoring during arthrographic evaluation of painful hip prostheses. American Journal of Roentgenology, 141(5):885-889.

Cooper, R. R., Milgram, J. W., and Robinson, R. A. (1966). Morphology of the osteon. J Bone Joint Surg Am, 48(7):1239-1271.

Cowin, S. (2000). Bone poroelasticity. In Cowin, S., editor, Bone biomechanics handbook, pages 23-1 23-31. CRC Press, 2nd edition.

Cowin, S. (2004). The plumbing of long bones. In Cerrolaza, M., Mart Âynez, G., DoblarẪl, M., and Calvo, B., editors, Computational bioengineering: current trends and applications, pages 45-67. Imperial College Press.

Donnelly, W., Kobayashi, A., Freeman, M., Chin, T., Yeo, H., West, M., and Scott, G. (1997). Radiological and survival comparison of four methods of fixation of a proximal femoral stem. Journal of Bone and Joint Surgery-British Volume, 79(3):351.

Dowd, J. E., Sychterz, C. J., Young, A. M., and Engh, C. A. (2000). Characterization of long-term femoral-head-penetration rates. association with and prediction of osteolysis. J Bone Joint Surg Am, 82-A(8):1102-7.

Elfick, A. P. D., Green, S. M., Krikler, S., and Unsworth, A. (2003). The nature and dissemination of uhmwpe wear debris retrieved from periprosthetic tissue of thr. Journal of Biomedical Materials Research Part A, 65A(1):95-108.

Fahlgren, A., Bostrom, M. P. G., Yang, X., Johansson, L., Edlund, U., Agholme, F., and Aspenberg, P. (2010). Fluid pressure and flow as a cause of bone resorption. Acta Orthopaedica, 81(4):508-516. 
Fluent, F. (2015). Section 18.4.1. ansys inc, canonsburg, pa.

Hendrix, R. W., Wixson, R. L., Rana, N. A., and Rogers, L. F. (1983). Arthrography after total hip arthroplasty: a modified technique used in the diagnosis of pain. Radiology, 148(3):647-52.

Hirakawa, K., Bauer, T. W., Stulberg, B. N., Wilde, A. H., and Secic, M. (1996). Characterization and comparison of wear debris from failed total hip implants of different types. Journal of Bone and Joint Surgery-American Volume, 78A(8):12351243.

Hui, P., Leung, P., and Sher, A. (1996). Fluid conductance of cancellous bone graft as a predictor for graft-host interface healing. Journal of biomechanics, 29(1):123-132.

Ise, K., Kawanabe, K., Matsusaki, T., Shimizu, M., Onishi, E., and Nakamura, T. (2007). Patient sensitivity to polyethylene particles with cemented total hip arthroplasty. J Arthroplasty, 22(7):966-73.

Jasty, M., Goetz, D. D., Bragdon, C. R., Lee, K. R., Hanson, A. E., Elder, J. R., and Harris, W. H. (1997). Wear of polyethylene acetabular components in total hip arthroplasty - an analysis of one hundred and twenty-eight components retrieved at autopsy or revision operations. Journal of Bone and Joint Surgery-American Volume, 79A(3):349-358.

Jell, G., Kerjaschki, D., Revell, P., and Al-Saffar, N. (2006). Lymphangiogenesis in the bone-implant interface of orthopedic implants: importance and consequence. Journal of Biomedical Materials Research Part A, 77A(1):119-127.

Johansson, L., Edlund, U., Fahlgren, A., and Aspenberg, P. (2009). Bone resorption induced by fluid flow. Journal of Biomechanical Engineering-Transactions of the Asme, 131(9):094505 (1-5).

Knothe, T. M. (2003). " whither flows the fluid in bone?" an osteocyte's perspective. Journal of Biomechanics, 36(10):1409-1424.

Kobayashi, A., Freeman, M. A. R., Bonfield, W., Kadoya, Y., Yamac, T., AlSaffar, N., Scott, G., and Revell, P. A. (1997). Number of polyethylene particles and osteolysis in total joint replacements - a quantitative study using a tissue-digestion method. Journal of Bone and Joint Surgery-British Volume, 79B(5):844-848.

Kohles, S., Roberts, J., Upton, M., Wilson, C., Bonassar, L., and Schlichting, A. (2001). Direct perfusion measurements of cancellous bone anisotropic permeability* 1. Journal of Biomechanics, 34(9):1197-1202. 
Koseki, H., Matsumoto, T., Ito, S., Doukawa, H., Enomoto, H., and Shindo, H. (2005). Analysis of polyethylene particles isolated from periprosthetic tissue of loosened hip arthroplasty and comparison with radiographic appearance. Journal of Orthopaedic Science, 10(3):284-290.

Kwong, L., Jasty, M., Mulroy, R., Maloney, W., Bragdon, C., and Harris, W. (1992). The histology of the radiolucent line. Journal of Bone and Joint Surgery-British Volume, 74(1):67.

Maloney, W. J., Jasty, M., Harris, W. H., Galante, J. O., and Callaghan, J. J. (1990). Endosteal erosion in association with stable uncemented femoral components. Journal of Bone and Joint Surgery, 72A(7):1025-1034.

Manley, M. T., D’Antonio, J. A., Capello, W. N., and Edidin, A. A. (2002). Osteolysis: a disease of access to fixation interfaces. Clin Orthop Relat Res, (405):129-37.

Margevicius, K., Bauer, T., McMahon, J., Brown, S., Merritt, K., et al. (1994). Isolation and characterization of debris in membranes around total joint prostheses. The Journal of bone and joint surgery. American volume, 76(11):1664.

Montgomery, R. J., Sutker, B. D., Bronk, J. T., Smith, S. R., and Kelly, P. J. (1988). Interstitial fluid flow in cortical bone. Microvascular research, 35(3):295-307.

Nauman, E. A., Fong, K. E., and Keaveny, T. M. (1999). Dependence of intertrabecular permeability on flow direction and anatomic site. Ann Biomed Eng, 27(4):517-24.

Noble, J., Jones, A. G., Davies, M. A., Sledge, C. B., Kramer, R. I., and Livni, E. (1983). Leakage of radioactive particle-systems from a synovial joint studied with a gamma-camera - its application to radiation synovectomy. Journal of Bone and Joint Surgery-American Volume, 65(3):381-389.

Pakula, M., Padilla, F., Laugier, P., and Kaczmarek, M. (2008). Application of biot's theory to ultrasonic characterization of human cancellous bones: Determination of structural, material, and mechanical properties. Journal of the Acoustical Society of America, 123(4):2415-2423.

Prendergast, P. J., Huiskes, R., and Soballe, K. (1997). Biophysical stimuli on cells during tissue differentiation at implant interfaces. Journal of Biomechanics, 30(6):539548.

Revell, P. A., Alsaffar, N., and Kobayashi, A. (1997). Biological reaction to debris in relation to joint prostheses. Proceedings of the Institution of Mechanical Engineers Part H-Journal of Engineering in Medicine, 211(2):187-197. 
Rhinelander, F. (1972). Circulation in bone. The biochemistry and physiology of bone, $2: 1-77$.

Robertsson, O., Wingstrand, H., Kesteris, U., Jonsson, K., and Onnerfalt, R. (1997). Intracapsular pressure and loosening of hip prostheses - preoperative measurements in 18 hips. Acta Orthopaedica Scandinavica, 68(3):231-234.

Schmalzried, T. P., Akizuki, K. H., Fedenko, A. N., and Mirra, J. (1997). The role of access of joint fluid to bone in periarticular osteolysis - a report of four cases. Journal of Bone and Joint Surgery-American Volume, 79A(3):447-452.

Schmalzried, T. P. and Callaghan, J. J. (1999). Wear in total hip and knee replacements. J Bone Joint Surg Am, 81(1):115-36.

Schmalzried, T. P., Jasty, M., and Harris, W. H. (1992). Periprosthetic bone loss in total hip arthroplasty. polyethylene wear debris and the concept of the effective joint space. Journal of Bone and Joint Surgery-American Volume, 74(6):849-63.

Shorez, J. P., Harding, T. S., Atkinson, P. J., and Walter, N. (2008). Alteration of the amount and morphology of wear particles by the addition of loading profile transitions during artificial hip wear testing. Proceedings of the Institution of Mechanical Engineers Part H-Journal of Engineering in Medicine, 222(H6):865-875.

Simkin, P., Pickerell, C., and Wallis, W. (1985). Hydraulic resistance in bones of the canine shoulder. Journal of biomechanics, 18(9):657-659.

Tanzer, M., Maloney, W. J., Jasty, M., and Harris, W. H. (1992). The progression of femoral cortical osteolysis in association with total hip-arthroplasty without cement. Journal of Bone and Joint Surgery-American Volume, 74A(3):404-410.

Thompson, M. S., Flivik, G., Juliusson, R., Odgaard, A., and Ryd, L. (2004). A comparison of structural and mechanical properties in cancellous bone from the femoral head and acetabulum. Proceedings of the Institution of Mechanical Engineers. Part H, Journal of Engineering in Medicine, 218(H6):425-429.

Urban, R. M., Jacobs, J. J., Tomlinson, M. J., Gavrilovic, J., Black, J., and Peoc'h, M. (2000). Dissemination of wear particles to the liver, spleen, and abdominal lymph nodes of patients with hip or knee replacement. Journal of Bone and Joint SurgeryAmerican Volume, 82A(4):457-477.

von Knoch, M., Engh, C. A., Sychterz, C. J., Engh, C. A., and Willert, H. G. (2000). Migration of polyethylene wear debris in one type of uncemented femoral component with circumferential porous coating - an autopsy study of 5 femurs. Journal of Arthroplasty, 15(1):72-78. 
${ }_{644}$ Willert, H. G., Bertram, H., and Buchhorn, G. H. (1990). Osteolysis in alloarthroplasty ${ }_{645}$ of the hip - the role of ultra-high-molecular-weight polyethylene wear particles. Clin${ }_{646} \quad$ ical Orthopaedics and Related Research, (258):95-107.

${ }_{647}$ Yuan X, ., Ryd, L., and Huiskes, R. (2000). Wear particle diffusion and tissue differen648 tiation in tka implant fibrous interfaces. Journal of Biomechanics, 33(10):1279-1286.

Zhang, D., Weinbaum, S., and Cowin, S. C. (1998). Estimates of the peak pressures in ${ }_{650}$ bone pore water. Journal of Biomechanical Engineering.Transactions of the Asme, $651 \quad 120(6): 697-703$.

Zicat, B., Engh, C. A., and Gokcen, E. (1995). Patterns of osteolysis around total hip components inserted with and without cement. Journal of Bone and Joint SurgeryAmerican Volume, 77A(3):432-439. 


\section{List of figure captions}

Figure 1: a) Realistic 3D model of an implanted femur and the surrounding joint capsule (left) and the 2D cross-sectional geometry generated based on coronal cut (right) . b) Schematic of the system consisting of the fluid capsule and gap regions in communication with the surrounding porous bone region. The size of the gap is exaggerated for demonstration purposes. Capsular pressure inlet (green) and periosteal and endosteal pressure outlets (blue) are shown.

Figure 2: The meshed geometry. It can be seen that mesh resolution is much higher in the gap region and its surrounding bone where there are large flow gradients in the model.

Figure 3: Particle concentration along a profile line which is located in the mid-gap region. This profile line is perpendicular to the interface and extends to up to the regions of farthest particle penetration. For ease of comparison its length is normalised. A gradual decrease in particle concentration occurs for $c_{f}=50 \%$.

Figure 4: Particle concentration in the bone. This schematic shows how osteolysis threshold can be calculated for smaller volumes of tissue using linear interpolation.

Figure 5: Contour plots of periprosthetic tissue showing simulated fibrous tissue generation in osteolytic lesions due to polyethylene particles for different values of $o s_{t}$. Fibrous tissue patterns tend to take a focal shape for a lower $o s_{t}$ and tend to be more linear for higher $o s_{t}$.

Figure 6: Fibrous tissue to bone ratio at the interface in proximal, middle and distal regions of the interfacial gap for different postoperative years.

\section{List of table captions}

Table 1: Simulation and solver setups in Fluent. The default Fluent under-relaxation factors for convergence were used. 